\title{
Efecto del Pretratamiento de Biomasa Maderera en el Rendimiento a Etanol
}

\author{
Elkin A. Gómez ${ }^{(1)}$, Luis A. Ríos ${ }^{(1) *}$ y Juan D. Peña ${ }^{(2)}$
}

(1) Depto. de Ingeniería Química, Facultad de Ingeniería, Universidad de Antioquia, Calle 70 No 52-21, Medellín-Colombia (e-mail: elkinandresgomez@yahoo.com; larios@udea.edu.co).

(2) Empresas Públicas de Medellín, E.S.P. Carrera 58 № 42-125, Medellín-Colombia

(e-mail: Juan.Pena.Alvarez@epm.com.co).

* Autor a quien debe ser dirigida la correspondencia.

Recibido Mar. 20, 2013; Aceptado Abr. 29, 2013; Versión final recibida May. 24, 2013

\begin{abstract}
Resumen
Muestras de madera de las especies Pinus patula y Eucalyptus camaldulensis fueron caracterizadas según procedimientos y protocolos establecidos por el Laboratorio Nacional de Energías Renovables de Estados Unidos, para determinar su composición. Posteriormente, astillas de estas maderas fueron pretratadas mediante explosión con vapor, deslignificación alcalina, remojo con amoniaco, ozonólisis, hidrólisis con ácido diluido y extracción con solvente orgánico para acondicionar el material crudo para la etapa de hidrólisis enzimática. Este paso fue llevado a cabo con el método enzimático Accellerase 1500 de Genecor seguida de fermentación del hidrolizado con la cepa Saccharomyces cerevisiae Ethanol Red mediante fermentación y sacarificación simultáneas. Los resultados de esta etapa permitieron seleccionar el mejor método de pretratamiento de las maderas. La deslignificación alcalina mostró los mejores rendimientos de etanol (2.17 g/L para Eucalyptus camaldulensis, y $2.61 \mathrm{~g} / \mathrm{L}$ para Pinus patula).
\end{abstract}

Palabras clave: biocombustibles, bioetanol, biomasa lignocelulósica, madera, pretratamiento

\section{Effect of Pretreatment of Woody Biomass on Ethanol Yield}

\begin{abstract}
Wood samples from Pinus patula and Eucalyptus camaldulensis species were characterized according to procedures and protocols established by the National Renewable Energy Laboratory (USA), to determine their composition. Then, chips from these woods were pretreated by steam explosion, alkaline delignification, ammonia wet soaking, ozonolysis, dilute acid hydrolysis and organic solvent techniques, to condition the raw materials for the enzymatic hydrolysis step. This step was carried out with the commercial enzymatic pull Accellerase 1500 from Genecor followed by fermentation of the hydrolyzate using Saccharomyces cerevisiae Ethanol Red yeast in a simultaneous saccharification and fermentation configuration. The results of this step allowed selecting the better pretreatment method for woods. Alkaline delignification showed the best ethanol yields $(2.17 \mathrm{~g} / \mathrm{L}$ for Eucalyptus camaldulensis, and $2.61 \mathrm{~g} / \mathrm{L}$ for Pinus patula).
\end{abstract}

Keywords: biofuels, bioethanol, lignocellulosic biomass, wood, pretreatment 


\section{INTRODUCCIÓN}

La problemática mundial que viene generando el carácter no renovable de los combustibles fósiles, su alta participación en el sector energético, la contaminación atmosférica de los centros urbanos, además de algunas preocupaciones en torno a la seguridad energética, ha impulsado fuertemente el desarrollo de procesos que usan materias primas renovables y fortalecido el interés en búsqueda de fuentes alternativas de energía diferentes a las petrolíferas para la obtención de combustibles. La biomasa es una fuente de energía primaria renovable y adecuada, ya que puede proporcionar algunas alternativas de combustibles para el transporte como el bioetanol, el biobutanol o el biodiesel en el corto plazo (Hamelinck et al., 2005; Sun y Cheng, 2002).

La producción actual de bioetanol se basa en etanol a partir de almidón y azúcares, pero esto ha generado un gran debate acerca de su sostenibilidad. En este contexto, el bioetanol producido a partir de biomasa lignocelulósica es una alternativa interesante, ya que las materias primas lignocelulósicas no compiten con los cultivos alimentarios y también son menos costosas que las materias primas agrícolas convencionales.

La biomasa lignocelulósica es la materia prima renovable más abundante, su producción anual se ha estimado $1 \times 10^{10}$ millones de toneladas alrededor del mundo (Sánchez et al., 2008). La conversión biológica de las diferentes materias primas lignocelulósicas, como lo son los bosques y los residuos agrícolas o los cultivos lignocelulósicos dedicados a la obtención de etanol ofrece numerosos beneficios, pero su desarrollo se ve obstaculizado por los factores económicos y técnicos (Sánchez et al., 2008). En este sentido, uno de los factores más importantes para reducir el costo de la producción de etanol son los siguientes: utilización eficiente de la materia prima para obtener altos rendimientos de etanol, una alta productividad, alta concentración de etanol en la destilación, la valorización de todos los coproductos y la integración de procesos con el fin de reducir la demanda de energía (Galbe y Zacchi, 2007).

En el Plan Energético Nacional colombiano (Unidad de Planeación Minero Energética, 2003) se establece la meta de aumentar el contenido de etanol en la gasolina, desde E10, que es el reglamentado actualmente, hasta mezclas E20 o E25 y se recomienda la producción excedente de etanol para exportación. Esto conduce a la necesidad de aumentar los cultivos y la capacidad instalada de la plantas de producción de etanol en Colombia, para lograr una producción de 25 millones de (L/día) de etanol en el 2025 a un precio competitivo con el mercado internacional.

Actualmente hay 50.120 ha dedicadas al cultivo de caña de azúcar para la producción de 948.061 (L/día) de etanol, lo que indica un rendimiento de 18,9 (L/(ha*día) y se están construyendo proyectos en la región del Pivijay en el Departamento de Magdalena y en Puerto López y Puerto Gaitán en el Departamento del Meta con una producción de 375.000 (L/día) en cada una, a partir de caña de azúcar, las cuales no son suficientes para lograr las metas del Plan Energético Nacional (Daza, 2011).

Las investigaciones colombianas en la producción de etanol están enfocadas en evaluar la producción de etanol a partir de diferentes fuentes de biomasa disponibles en el país, tales como bagazo de caña, cascarilla de café, residuos de banano y de palma. Es por esta razón que se presenta en este artículo una alternativa, investigando el efecto de diferentes pretratamientos sobre material maderero proveniente de Guarne, Antioquia (Colombia), con el objeto de producir etanol a partir de su fracción celulósica.

\section{METODOLOGÍA}

El material maderero contiene aproximadamente $40-50 \%$ de celulosa, $25-35 \%$ de hemicelulosa y $20-35 \%$ de lignina y son la principal fuente de material lignocelulósico en Colombia (Gómez et al., 2012). Normalmente, los residuos de la industria maderera son incinerados para generación de energía y calefacción, o son dispuestos finalmente como basura, siendo una fuente potencial de biomasa renovable para producir etanol. Las alternativas de aprovechamiento implementadas hasta el momento se han enfocado fundamentalmente hacia su recuperación energética (Varnero et al., 2010).

Dado que para fermentar los azúcares presentes en la biomasa es necesario romper la estructura lignocelulósica protegida principalmente por la lignina, se requiere entonces de un proceso de acondicionamiento de la materia prima. Muchos son las técnicas de pretratamiento que se han empleado para acondicionar la biomasa lignocelulósica, pero pocas técnicas se han empleado con materiales madereros, puesto que éstos son los que ofrecen mayor resistencia estructural dado su alto contenido de lignina. Entre las técnicas más usadas se encuentran el tratamiento con solventes orgánicos, la explosión con vapor, la deslignificación alcalina y la hidrólisis ácida diluida.

Si bien el contenido de hemicelulosa es lo suficientemente alto como para considerarse una fracción importante a la hora de realizar un aprovechamiento apropiado del material maderero, las dificultades a la 
hora de fermentar esta fracción sugieren que el tema sea objeto de estudio en posteriores investigaciones. Algunos autores han reportado problemas de tipo inhibitorio a la hora de fermentar pentosas por efectos de los productos de degradación de los azúcares en la etapa de pretratamiento, por concentración de los mismos azúcares y por la presencia de los mismos productos de la fermentación (Agbogbo y Coward-Kelly, 2008; Almeida et al., 2011; Zhang y Geng, 2012).

Ewanick et al. (2007) pretrataron astillas del pino Lodgepole mediante explosión con vapor a temperaturas entre 195 y $215^{\circ} \mathrm{C}$, con tiempos de residencia de hasta 5 min y concentración de catalizador ácido de $4 \%$ obteniendo recuperación de glucosa hasta del 41\%. Ballesteros et al. (2000) obtuvo recuperaciones de azúcares totales del orden de $47,8 \%$ al tratar astillas de pino Pinaster por explosión con vapor a $210^{\circ} \mathrm{C}$ por 8 min. Pero los resultados más positivos son los obtenidos por Söderström et al. (2004), quien pretrató astillas de picea mediante explosión con vapor a $215^{\circ} \mathrm{C}$ por $5 \mathrm{~min}$, usando como catalizador $\mathrm{SO}_{2}$ al $3 \%$. Bajo estas condiciones logró recuperar el $71 \%$ de los azúcares en la materia prima.

Dada la gran extensión en la cobertura forestal del territorio colombiano, se hace oportuna la investigación en el campo del aprovechamiento del recurso lignocelulósico maderero. Colombia no solo cuenta con una gran área sembrada de especies forestales comerciales, sino que además cuenta con una industria maderera bien desarrollada, que produce buena cantidad de residuos madereros al año. Una de las aplicaciones con mayor atractivo actualmente es el de los biocombustibles. Por estas razones, se expone en este artículo una primera aproximación a las técnicas de pretratamiento de material maderero a ser empleado en la producción de bioetanol.

Dentro de las etapas comprendidas para la conversión biológica de biomasa a etanol, el pretratamiento de la biomasa es la segunda etapa más cara en el costo de la conversión de biomasa lignocelulósica en etanol mediante hidrólisis enzimática, precedido por los costos de la materia prima (Mosier et al., 2005).

Como primer acercamiento al tema se buscó hacer una evaluación cualitativa de las especies madereras más profusas en Colombia, que incluyó entre otras, Cupresus lusitanica, Eucalyptus globulus, Eucalyptus grandis, Pinus caribaea, Pinus radiata, etc. Evaluando rendimiento volumétrico anual, condiciones edafológicas y climáticas de cultivo, composición química aproximada y hectáreas disponibles, se obtuvieron como especies más idóneas el Pinus patula y el Eucalyptus camaldulensis para ser empleadas en la producción de etanol carburante (Gómez et al., 2012). Estas especies se caracterizan por tener rendimientos volumétricos elevados de entre 20 y 30 ( $\mathrm{m}^{3} /\left(\mathrm{ha}^{\star}\right.$ año) (Kropff, 2000; Gillespie, 2004), y contenidos de celulosa y hemicelulosa de hasta 50 y 30\%, respectivamente (Kropff, 2000; Uribe, 1967). Otro punto clave fue el contenido de lignina, de los más bajos entre las especies evaluadas (Kropff, 2000; Uribe, 1967).

\section{DESARROLLO EXPERIMENTAL}

\section{Materia prima}

Astillas de madera de Pinus patula y Eucalyptus camaldulensis, libres de corteza fueron provistas por la industria maderera de Guarne, Antioquia.

\section{Reducción de tamaño}

Antes que la fracción de celulosa de la madera pueda usarse como sustrato para la producción de etanol, debe ser separada de su envoltura de hemicelulosa y lignina que la protege y debe extenderse el área superficial efectiva disponible para los microorganismos. La reducción de la madera a un tamaño compatible con el proceso subsecuente es el primer paso del pretratamiento. La reducción de tamaño prácticamente no agrega valor al material crudo, tiene altos requerimientos de energía y puede incrementar significativamente los costos de producción. La disminución de los materiales lignocelulósicos a través de una combinación de astillado y/o molienda puede aplicarse para reducir la cristalinidad de la celulosa. El tamaño de los materiales suele ser de 10-30 mm después del astillado y de 0,2-2 mm después de la molienda (Sung et al., 2002).

Monavari et al. (2009) estudiaron el rendimiento de azúcares para diferentes tamaños de partículas de astillas de picea (espesores de 1-2 mm, 3-4 mm y 5-6 mm y longitudes promedio de 30-45 mm) pretratadas con explosión de vapor y $\mathrm{SO}_{2}$ con tiempos de impregnación variables. El rendimiento global más alto de glucosa y manosa fue de $73 \%$, y se obtuvo para los tamaños de partícula de 1-2 mm, mientras que el rendimiento más bajo fue de $69 \%$ para astillas de 5-6 $\mathrm{mm}$. 
Las astillas procedentes de aserraderos locales se trataron con una picadora vegetal de cuchillas hasta alcanzar un tamaño entre $10 \mathrm{~mm}$ y $30 \mathrm{~mm}$, tamaño que si bien no destruye la cristalinidad de la celulosa, sí facilita el acceso del pull enzimático al sustrato en el proceso de hidrólisis (Sung y Cheng, 2002). Se realizó el análisis granulométrico de los materiales secos utilizando el método de análisis mecánico, mediante tamices de abertura a cada uno de los materiales, de acuerdo a las normas ASTM D422 y NTC 1522. Los equipos usados fueron tamices estándares de la serie ASTM Pinzuar, un rotap y una balanza de precisión. Las mallas se escogieron de acuerdo a los tamaños mayores y menores de los sólidos manipulados, en este caso se usaron las mallas: $1 / 4,4,10,20,35,45,60$ y 80 que abarcan tamaños entre 6350 y 150 micras.

\section{Explosión con vapor}

Las variables importantes en el pretratamiento de explosión con vapor incluyen el tiempo, la temperatura, el tamaño de las astillas y el contenido de humedad (Saddler et al., 1993). Algunos autores han profundizado en la investigación del efecto de cada una de estas variables en el pretratamiento de material maderero a ser usado para producir etanol (Ewanick et al., 2007; Ballesteros et al., 2000; Söderström et al., 2004; Monavari et al., 2009). Puesto que el principal inconveniente con esta tecnología ha sido el efecto de los inhibidores procedentes de la descomposición del material lignocelulósico, se decidió operar el explosionador de vapor a condiciones similares a las reportadas en el trabajo de Söderström et al. (2004) disminuyendo el tiempo de residencia de $5 \mathrm{~min}$ a $2 \mathrm{~min}$ y fijando temperatura en $210^{\circ} \mathrm{C}$ y presión en 1,9 MPa. Por factibilidad técnica se empleó $\mathrm{H}_{2} \mathrm{SO}_{4}$ al $0,175 \%$ en lugar de $\mathrm{SO}_{2}$ al $3 \%$. Con estas condiciones, la severidad, Log (R0), resultante para el experimento quedó estalecida en 3,54. La relación sólido líquido quedó establecida en 3:40 debido a limitaciones técnicas del equipo explosionador empleado.

La materia prima molida dispuesta en bandejas metálicas se impregnó con ácido sulfúrico diluido al 0,175\% mediante spray hasta alcanzar un valor de humidificación del 30\%. Las muestras se secaron al aire libre por 24 horas y se dispusieron en bolsas plásticas.

El pretratamiento mediante explosión con vapor se efectuó en el equipo explosionador con vapor DIES S.A. perteneciente al Grupo Pulpa y Papel de la Universidad Pontificia Bolivariana. El equipo genera su propio vapor, por lo que el material procesado está sujeto a una etapa lenta de calentamiento y presurización hasta que se alcanzan las condiciones de presión y temperatura requeridas. Para alcanzar la condición de severidad establecida, se elimina el calentamiento al conseguir la temperatura y se procede a contabilizar el tiempo, al término del cual, se detiene el proceso. El procesamiento de la materia prima se llevó a cabo cargando el material $(150 \mathrm{~g})$ directamente en el contenedor de muestras del equipo y adicionando luego $2 \mathrm{~L}$ de agua líquida en el compartimiento de evaporación, . Después de alcanzar el valor requerido de severidad Log (R0), la carga de biomasa/vapor fue liberada mediante una despresurización rápida del tanque de presión del equipo, accionando una válvula de bola, provocando la expansión del material en un tanque de mayor volumen. Finalmente se recogió el hidrolizado en recipientes de $2 \mathrm{~L}$ y se recuperó el sustrato, lavándose con abundante agua para ser almacenado en bolsas plásticas.

\section{Remojo con amoniaco acuoso}

Este pretratamiento, consiste en una deslignificación alcalina bajo condiciones moderadas, ya que se emplean bajas temperaturas y presión atmosférica, pero a cambio requiere tiempos de residencia mucho más grandes que cuando se emplean condiciones más severas de presión y temperatura.

Este pretratamiento consiste en el remojo del material, con una solución acuosa de amoniaco al $15 \%$ (w/w), con una relación sólido líquido de 1:6. El remojo de los materiales se lleva a cabo a $60^{\circ} \mathrm{C}$ durante 6 horas (Kim y Lee, 2007; Li et al., 2010), tiempo después del cual la reacción se detiene súbitamente enfriando el reactor en un baño de hielo, y posteriormente se filtra para separar las fracciones sólida y líquida. La fracción sólida se neutraliza y se lava para retirar el licor negro remanente, y se guarda para los procesos siguientes de hidrólisis y fermentación.

Deslignificación alcalina con $\mathrm{NaOH}$

Esta técnica solubiliza la lignina para separar la fracción fermentable de la no fermentable del material lignocelulósico maderero, al tiempo que rompe la rigidez de la estructura para permitir un mejor acceso a la matriz celulósica y hemicelulósica en la etapa de hidrólisis.

El pretratamiento es realizado en un reactor de acero inoxidable con recubrimiento interno de teflón, el cual está diseñado para soportar la presión interna que se genera al calentar la mezcla a la temperatura indicada. Como sistema de calentamiento se usó una estufa de convección dotada de control de temperatura, así como de temporizador para controlar el tiempo de calentamiento. 
Los valores de temperatura, tiempo y demás fueron obtenidos de literatura, en particular se tomaron los reportados por Gupta (2008), acondicionándolos para que fuesen aplicables a todos los materiales seleccionados y para que sirviesen de criterio para decidir si el pretratamiento fue efectivo o no, tomando como respuesta la fermentabilidad del material hidrolizado. Se tuvo también en cuenta la rigurosidad del pretratamiento, pues condiciones muy extremas si bien aumentan la deslignificación ocasionan pérdidas de hemicelulosa, lo que reduce la cantidad de azúcares fermentables.

El pretratamiento se llevó a cabo a una temperatura de $120^{\circ} \mathrm{C}$, presión de $200 \mathrm{KPa}$, relación sólido líquido de $1 / 15$, concentración de $\mathrm{NaOH}$ de $1 \%$ en peso por $30 \mathrm{~min}$.

Se pesó la cantidad de material (aproximadamente $20 \mathrm{~g}$ por experimento), se llevó al reactor y una vez allí se le adicionó la cantidad indicada de solución de $\mathrm{NaOH}$, luego se cerró el reactor herméticamente y se llevó a la estufa, ésta se programó para realizar un calentamiento lento hasta los $120^{\circ} \mathrm{C}$ (aproximadamente en 15 minutos) y luego se dejó en esa temperatura por 30 minutos. Completado el tiempo se enfrió hasta temperatura ambiente, para luego filtrar para separar el material del licor negro. El sólido se lavó con agua hasta $\mathrm{pH}$ neutro para luego secarlo y almacenarlo en nevera para su posterior uso.

\section{Organosolv}

Existe una amplia gama de solventes que han sido utilizados y estudiados en el fraccionamiento con solventes de los materiales lignocelulósicos. Sin embargo la dificultad y los costos elevados de la recuperación de éstos hacen que la practicidad de su empleo disminuya considerablemente. Araque et al. (2008) optimizaron las condiciones del pretratamiento organosolv con acetona para pretratar astillas de Pinus radiata D. Don y conseguir un rendimiento de etanol elevado. El material pretratado a una temperatura de $185^{\circ} \mathrm{C}$, por $5 \mathrm{~min}$, con relación acetona: agua de 1:1 produjo un rendimiento de etanol del $99,5 \%$. En la parte experimental que se efectuará de este pretratamiento, se emplearán estas condiciones dada la naturaleza similar de las materias primas.

El pretratamiento se llevó a cabo en un reactor de 0,5 L de acero inoxidable cargado con $50 \mathrm{~g}$ de astillas de madera y $350 \mathrm{~mL}$ de solución acuosa al 50\% v/v de acetona conteniendo ácido sulfúrico como catalizador (0,9\% w/w base madera seca). La relación solvente: madera dentro del reactor fue de 7:1. Después de sellar el reactor, ése se llevó a una estufa en la que se calentó por 3 min a $185^{\circ} \mathrm{C}$. Al completarse este tiempo, el reactor se enfrió con baño de hielo para acelerar la condensación del vapor. Finalmente se separó la fase sólida de la líquida mediante filtración al vacío y se procedió con el almacenamiento del hidrolizado en frascos limpios y del sustrato en bolsas plásticas a una temperatura de $4^{\circ} \mathrm{C}$.

\section{Ozonólisis}

El ozono ha sido utilizado para degradar la lignina y la hemicelulosa de numerosos materiales lignocelulósicos como paja de trigo, bagazo, pino, algodón y serrín de chopo (Vidal y Molinier, 1988). En las décadas recientes, el pretratamiento con ozonólisis ha demostrado su eficacia esencialmente en la degradación de la lignina, dada la alta reactividad del ozono hacia compuestos que incorporan dobles enlaces conjugados, pero también solubilizando ligeramente el contenido de hemicelulosa en la biomasa lignocelulósica y dejando intacta la celulosa (Sun y Cheng, 2002). Las principales ventajas vinculadas a este proceso son la ausencia de productos de degradación que pudieran interferir con la posterior hidrólisis o fermentación y que las reacciones se producen a temperatura ambiente (Vidal y Molinier, 1988). Silverstein et al. (2007), aplicó recientemente pretratamiento de ozono para la conversión de tallos de algodón a etanol por aspersión continua de gas de ozono a través de una mezcla de tallos de algodón y agua 10\% (w / v) a 4 ${ }^{\circ} \mathrm{C}$ durante 30, 60 y 90 minutos. En este estudio, el pretratamiento con ozono reduce la lignina en el rango de 11,97 a $16,6 \%$ sin diferencias significativas notadas en los tiempos de tratamiento, de 30,60 y 90 min.

Este pretratamiento se llevó a cabo a temperatura ambiente y una relación sólido líquido de 1:6, condiciones reportadas por Vidal y Molinier (1988). El flujo de ozono para este pretratamiento se fijó de acuerdo a la capacidad y disponibilidad del equipo de generación de ozono. El ozono es generado a partir de oxígeno empleando un flujo de $1 \mathrm{l} / \mathrm{min}$ de dicho gas.

El sistema de pretratamiento con ozono consiste en hacer burbujear el ozono a través del material, donde éste se encarga de degradar la lignina. El tiempo de reacción empleado fue de 60 minutos y el flujo de ozono fue de $5,4 \mathrm{mg}$ de ozono/minuto. La cantidad de ozono suministrado y la cantidad de ozono consumido por la reacción se determinó mediante técnicas analíticas volumétricas, absorbiendo el ozono en una solución de yoduro de potasio al 2\% (w/w), y posteriormente titulando la solución con tiosulfato de sodio 0,005 $\mathrm{N}$ después de añadir $10 \mathrm{ml}$ de ácido sulfúrico $2 \mathrm{~N}$ a la solución de yoduro de potasio. 


\section{Hidrólisis ácida diluida}

La hidrólisis ácida de las astillas de madera se llevó a cabo bajo las siguientes condiciones seleccionadas entre las reportadas en la literatura: tiempo de residencia de 90 minutos, temperatura de $90^{\circ} \mathrm{C}$, ácido sulfúrico en concentración de 2\% (w/w), relación sólido líquido 1:10 (Alvira et al., 2010; Cardona y Sánchez, 2007; Dien et al., 2006; Silverstein et al., 2007). El material lignocelulósico es pesado (aproximadamente $100.0 \mathrm{~g}$ ) y depositado en un reactor y se adiciona la solución de ácido sulfúrico de acuerdo a la relación sólido/líquido, la suspensión se agita mecánicamente a la temperatura fijada por el tiempo de 90 minutos. Después de transcurrido el tiempo de reacción, el reactor se enfría súbitamente para detener las reacciones que se dan durante el proceso. Luego la suspensión se filtra para separar el licor negro del material pretratado, el material se lava para retirar el licor negro remanente y se neutraliza para las etapas siguientes de hidrólisis y fermentación.

\section{Caracterización de las materias primas y de los materiales lignocelulósicos pretratados}

La caracterización fisicoquímica de la madera pretratada de Pinus patula y Eucalyptus camaldulensis se llevó a cabo siguiendo los protocolos para la caracterización de biomasa del National Renewable Energy Laboratory (NREL) e incluyó determinación de cenizas, de extraíbles, carbohidratos estructurales y lignina en biomasa. El contenido de azúcares reductores en las muestras hidrolizadas se determinó utilizando el método del ácido 3,5-dinitrosalicílico (DNS) con glucosa como estándar (Marsden et al., 1982; Miller, 1959). La determinación del contenido de etanol en los caldos de fermentación se llevó a cabo mediante la técnica SPME en un espectrómetro CG-FID empleando la columna de alta resolución HP Innowax 19091N-233.

\section{Sacarificación y fermentación simultáneas (SSF)}

Antes de medir y comparar los efectos de los diferentes pretratamientos (explosión con vapor, hidrólisis ácida diluida, deslignificación con $\mathrm{NaOH}$, remojo con amoniaco acuoso, organosolv y ozonólisis) en las maderas de las especies de pino y eucalipto y seleccionar el mejor para cada una, se llevó a cabo una sacarificación y fermentación simultáneas (SSF). Esta configuración, en la que el pull enzimático y la levadura trabajan simultáneamente, se seleccionó debido a que de esta forma, se evitan acumulaciones de la fuente de carbono que puedan inhibir la levadura, pues al tiempo que la celulasa hidroliza el material, la levadura consume los azúcares para su crecimiento, mantenimiento y/o producción de etanol. Igualmente, se aumenta el rendimiento en comparación con la hidrólisis y fermentación separadas, pues aunque se tengan que alterar un poco las condiciones óptimas como temperatura y agitación de cada una de las fases, el sistema separado puede producir inhibición de la levadura por productos de la hidrólisis, el tiempo del proceso es más largo y existe una mayor probabilidad de contaminación. (Mejía y Alban, 2009).

Para llevar a cabo la sacarificación y fermentación simultáneas, se empleó el complejo enzimático de celulasas Accellerase 1500 de Genecor y la levadura comercial Saccharomyces cerevisiae Ethanol Red. En estos experimentos, se esterilizó el material lignocelulósico a 0.5 atm por $15 \mathrm{~min}$. Para la hidrólisis se cargaron $30 \mathrm{FPU} / \mathrm{g}$ y se montaron los sistemas en un shaker a una temperatura de $50^{\circ} \mathrm{C}$ (condiciones recomendadas por Genecor), $150 \mathrm{rpm}$ y buffer citrato $0.05 \mathrm{M}, \mathrm{pH} 4.8$ para amortiguar cambios en acidez 0 alcalinidad del sistema. Simultáneamente, transcurridas 12 horas, se inocula la levadura ( $2 \mathrm{~g} / \mathrm{L})$ bajando la temperatura a $37^{\circ} \mathrm{C}$ y aumentando la agitación a $180 \mathrm{rpm}$. En esta fase, al erlenmeyer se le acopló un respirómetro, en el cual se asegura que el $\mathrm{CO}_{2}$ liberado de la reacción se disuelva en la trampa de agua. Se realizó el monitoreo al sistema cada 2 horas durante 72 horas.

\section{RESULTADOS Y DISCUSIÓN}

\section{Granulometría del material}

El análisis granulométrico mostró que más del $90 \%$ del material de $P$. patula tiene un tamaño por debajo de los $3 \mathrm{~mm}$. Por el contrario, solo el $60 \%$ de la madera del eucalipto mostró un tamaño de partícula por debajo de los $3 \mathrm{~mm}$, tamaños de partícula similares a los reortados en la literatura como los más apropiados para hidrolizar la materia prima (Sung et al., 2002; Monavari et al., 2009).

\section{Caracterización de las materias primas}

La caracterización fisicoquímica de la madera de $P$. patula y E. camaldulensis se llevó a cabo siguiendo los protocolos para la caracterización de biomasa del National Renewable Energy Laboratory (NREL) e incluyó determinación de cenizas, de extraíbles, carbohidratos estructurales y lignina. La composición del material se presenta en la tabla 1 . Aproximadamente, $60 \%$ de la biomasa proveniente de la madera del pino y del eucalipto podría emplearse en la producción de etanol, en caso de aprovecharse la fracción hemicelulósica. 
Los datos que se muestran en las tablas corresponden al valor promedio de experimentos por triplicado con una desviación estándar relativa menor del $5 \%$ en todos los casos.

Tabla 1: Composición de madera (\%) de Pinus patula y Eucalyptus camaldulensis.

\begin{tabular}{|c|c|c|}
\hline Materia prima & $\begin{array}{c}\text { Eucalyptus } \\
\text { camaldulensis }\end{array}$ & Pinus patula \\
\hline Celulosa & 45,03 & 36,64 \\
\hline Hemicelulosa & 17,90 & 24,97 \\
\hline Lignina & 29,45 & 28,53 \\
\hline Ceniza & 1,11 & 0,28 \\
\hline Extraíbles & 1,93 & 1,50 \\
\hline Humedad & 5,48 & 7,21 \\
\hline
\end{tabular}

Si bien no existen antecedentes en Colombia en la caracterización química de la madera de esta especie de eucalipto, pueden compararse los resultados a la luz de los reportados internacionalmente. Kropff (2000) obtuvo contenidos de celulosa, hemicelulosa y lignina entre $41-50 \%, 14-19 \%$ y $18-34 \%$, respectivamente, para variedades de $E$. camaldulensis en el continente africano, lo que refuerza la veracidad de los resultados obtenidos en la presente investigación. Por otro lado, Uribe (1967) obtuvo 48,75\%, 32,75\% y $18,5 \%$ para los contenidos de celulosa, hemicelulosa y lignina, respectivamente, para la especie $P$. patula en la caracterización de materia prima a ser empleada en la fabricación de pulpa para papel. Aunque estos últimos resultados fueron obtenidos en la caracterización de madera local, obtenida del mismo sector del que se tomaron las muestras actuales, las diferencias se deben a que la composición de la madera de una especie forestal no puede determinarse de manera global, ya que varía con la parte del árbol de la que se tome la muestra que se analiza (raíz, tallo, hojas), la ubicación geográfica de la especia forestal, el clima y las condiciones del suelo bajo las que se ha desarrollado (Pettersen, 1984). Es por esta razón que análisis composicionales no pueden usarse para identificar especies arbóreas individuales dadas las variaciones dentro de cada especie y las similitudes entre muchas. Dado que los contenidos de lignina son muy similares para ambas especies, puede concluirse que la especie más apta para ser usada en la producción de etanol celulósico es el eucalipto, puesto que se caracteriza por el más alto contenido de celulosa en su estructura, esto, sin realizar ninguna valoración de la estructura lignocelulósica de su madera.

\section{Evaluación de pretratamientos}

Los pretratamientos produjeron dos fases, una fase sólida, rica en celulosa y una fase líquida, licor negro, constituido principalmente por lignina, ambas fracciones, sólida y líquida conteniendo hemicelulosas. Dado que el objetivo de esta investigación era la fermentación de la fracción celulósica, se procedió a realizar lavados y a neutralizar el $\mathrm{pH}$ de la fracción sólida. La caracterización de la fracción líquida no se incluye dado que la fermentación de hemicelulosas y el aprovechamiento de lignina es tema de otra investigación paralela.

El primer criterio empleado en la medición de la efectividad del pretratamiento es el contenido de azúcares fermentables. El contenido de azúcares reductores de las maderas pretratadas mediante las diferentes técnicas se muestra en la Tabla 2. La mayor liberación de azúcares reductores en la hidrólisis de la madera del eucalipto se obtuvo con los pretratamientos remojo con amoniaco, Organosolv y explosión con vapor, con resultados entre 25,6 y $28,1 \mathrm{mg}$ azúcares/g biomasa seca, resultados muy similares a los obtenidos en la hidrólisis de la madera de pino pretratada mediante extracción organosolv y explosión con vapor, 28.1 y 29,2 mg azúcares/g biomasa seca, respectivamente.

Los montajes de fermentabilidad arrojaron los resultados listados en la Tabla 3. En este caso, los pretratamientos mostraron un desempeño algo diferente entre sí. La producción de etanol fue más elevada para el caso de la madera de eucalipto pretratada mediante deslignificación alcalina, extracción Organosolv y explosión de vapor. 
Tabla 2: Concentración azúcares reductores en maderas pretratadas.

\begin{tabular}{|c|c|c|}
\hline \multicolumn{2}{|c|}{ Azúcares reductores g/L, (mg azúcares/g biomasa seca) } \\
\hline Pretratamiento & $\begin{array}{c}\text { Eucalyptus } \\
\text { camaldulensis }\end{array}$ & Pinus patula \\
\hline Ozonólisis & $2,56(13,77)$ & $4,62(24,85)$ \\
\hline Remojo con amoniaco & $4,80(25,82)$ & $3,20(17,21)$ \\
\hline Deslignificación alcalina & $4,34(23,35)$ & $4,14(22,27)$ \\
\hline Hidrólisis ácido diluido & $3,96(21,30)$ & $3,58(19,26)$ \\
\hline Organosolv & $5,22(28,08)$ & $5,22(28,08)$ \\
\hline Explosión con vapor & $4,76(25,61)$ & $5,42(29,16)$ \\
\hline
\end{tabular}

Tabla 3: Resultados SSF en maderas pretratadas.

\begin{tabular}{|c|c|c|}
\hline \multicolumn{3}{|c|}{ Rendimiento etanol $\mathrm{g} / \mathrm{L},(\mathrm{mg}$ etanol/g biomasa seca) } \\
\hline Pretratamiento & $\begin{array}{c}\text { Eucalyptus } \\
\text { camaldulensis }\end{array}$ & Pinus patula \\
\hline Ozonólisis & $1,23(7,39)$ & $1,40(8,39)$ \\
\hline Remojo con amoniaco & $1,28(7,66)$ & $1,52(9,12)$ \\
\hline Deslignificación alcalina & $2,18(13,05)$ & $2,61(15,65)$ \\
\hline Hidrólisis ácido diluido & $1,05(6,32)$ & $2,57(15,44)$ \\
\hline Organosolv & $1,92(11,52)$ & $1,95(11,71)$ \\
\hline Explosión con vapor & $1,92(11,52)$ & $1,30(7,79)$ \\
\hline
\end{tabular}

Contrario a lo que se esperaba, la producción de bioetanol para la madera de eucalipto pretratada mediante remojo con amoniaco fue de las menores sin importar que dicho sustrato haya producido uno de los contenidos de azúcares reductores más elevados. Para el caso de la madera de pino, la productividad alcohólica fue mayor para el material pretratado con deslignificación alcalina, hidrólisis ácida y Organosolv. En ambas maderas, el pretratamiento de mejor desempeño fue la deslignificación alcalina, pues arrojó fermentabilidades por encima de 2,18 g/L, Los rendimientos de etanol fueron muy similares a los obtenidos por Nguyen et al. (1998). Nguyen et al. (1998) obtuvo concentraciones de etanol de 2,5 g/L, pretratando madera de abeto Douglas y pino Ponderosa a $201^{\circ} \mathrm{C}$, por $125 \mathrm{~s}$, usando impregnación con $\mathrm{H}_{2} \mathrm{SO}_{4}$ al $0,4 \%$, relación sólido - líquido de 1:20.

Los resultados obtenidos indican que la deslignificación alcalina, la extracción con solventes orgánicos organosolv, la explosión con vapor y la hidrólisis ácida diluida permiten una mayor bioconversión de los materiales madereros del $P$. patula y $E$. camaldulensis a etanol.

\section{CONCLUSIONES}

De los resultados obtenidos en la evaluación del efecto del pretratamiento en biomasa maderera de Eucalyptus camaldulensis, puede concluirse que la deslignificación alcalina, la explosión con vapor y la extracción con solventes orgánicos han probado ser las técnicas más efectivas para acondicionar el sustrato a ser empleado en la producción de bioetanol carburante. En el acondicionamiento de la biomasa maderera del Pinus patula, las técnicas de deslignificación alcalina, hidrólisis con ácido diluido y extracción organosolv probaron ser las más efectivas, arrojando ambas resultados similares en productividad de bioetanol. También se concluye que, aunque el sustrato resultante del pretratamiento de remojo con amoniaco rinde altos contenidos de azúcares reductores, la productividad de la fermentación realizada sobre ellos no fue 
particularmente alta, hecho que podría explicarse por la presencia considerable de inhibidores formados durante la etapa de acondicionamiento. Mención especial merece el caso de la deslignificación alcalina, pretratamiento que, si bien, no se caracterizó por sus rendimientos de azucares reductores, estuvo a la par en productividad de etanol con las técnicas Organoslv, explosión con vapor e hidrólisis ácida diluida, indicando que es una técnica de bajo impacto en cuanto a presencia de inhibidores se refiere.

Las técnicas de pretratamiento que emplean ozono y amoniaco, si bien arrojaron resultados aceptables, presentan serias dificultades técnicas, dada la necesidad de manipular concentraciones relativamente altas de amoniaco y la necesidad de producir grandes cantidades de ozono para efectuar el pretratamiento. Puesto que existe una gran variabilidad en las características de la biomasa maderera local y la foránea, es recomendable la experimentación que permita una optimización de estas técnicas de pretratamiento para las especies nativas colombianas. Sin embargo, a la luz de los objetivos propuestos en el presente trabajo, se ha logrado hacer una evaluación apropiada de los pretratamientos con un alcance que permite direccionar la investigación futura.

El estudio en el campo de combustibles verdes es altamente recomendado, dado el gran beneficio extraíble de los recursos madereros disponibles en Colombia. Por otro lado, cada día se innova en el desarrollo de nuevas técnicas de pretratamiento y, por esta razón, se hace necesario el estudio de éstas en los materiales disponibles localmente. El costo del pretratamiento es un aspecto de gran importancia dentro del proceso, sin embargo, consideramos que es un aspecto que debe evaluarse de acuerdo no sólo al rendimiento de celulosa y hemicelulosa, sino también con los rendimientos obtenidos después del proceso de hidrólisis y fermentación, por lo tanto dependerá de la evaluación de cada pretratamiento para cada material.

\section{REFERENCIAS}

Agbogbo, F. K. y G. Coward-Kelly, Cellulosic ethanol production using the naturally occurring xylosefermenting yeast, Pichia stipitis, Biotechnology letters, 30(9), 1515-24 (2008).

Almeida, J. R. M., D. Runquist, V. Sànchez i Nogué, G. Lidén y M.F. Gorwa-Grauslund, Stress-related challenges in pentose fermentation to ethanol by the yeast Saccharomyces cerevisiae, Biotechnology journal, 6(3), 286-99 (2011).

Alvira, P., M. Ballesteros y M.J. Negro, Pretreatment technologies for an efficient bioethanol production process based on enzymatic hydrolysis: A review, Bioresource Technology, 101(13), 4851-4861 (2010).

Araque, E., C. Parra, J. Freer, D. Contreras, J. Rodríguez, R. MendonÇa y J. Baeza, Evaluation of organosolv pretreatment for the conversión of Pinus radiata D. Don to ethanol, Enzyme and Microbial Technology 43 (2008) 214-219 (2008).

Ballesteros, I., J. M. Oliva, A. A. Navarro, A. Gonzalez, J. Carrasco, M. Ballesteros, Effect of chip size on steam explosion pretreatment of softwood, Applied Biochemistry and Biotechnology 84-86, 97-110 (2000).

Cardona, C. A. y O. J. Sánchez, Fuel ethanol production: process design trends and integration opportunities, Bioresour. Technol., 98, 2415-2457 (2007).

Daza, C. Federación Nacional de Biocombustibles de Colombia (2011).

Dien, B. S., H.G. Jung, K.P. Vogel, M.D. Casler, J. F. S. Lamb, L. Iten, R. B. Mitchell, G. Sarath, Chemical composition and response to dilute-acid pretreatment and enzymatic saccharification of alfalfa, reed canarygrass, and Switchgrass, Biomass and Bioenergy, 30, 880-891 (2006).

Ewanick, S.M., R. Bura, J. N. Saddler, Acid-catalyzed steam pretreatment of lodgepole pine and subsequent enzymatic hydrolysis and fermentation to ethanol, Biotechnology and Bioengineering 98, 737-746 (2007).

Galbe, M. y G. Zacchi, Pretreatment of lignocellulosic materials for efficient bioethanol production, Adv. Biochem. Eng./Biotechnol., 108, 41-65 (2007).

Gillespie, A. Pinus patula Schiede \& Deppe. Pino pátula, ocote. International Institute of Tropical Forestry (IITF). United States Department of Agriculture (USDA), Forest Service.

http://www.fs.fed.us/global/iitf/Pinuspatula.pdf (2004). Acceso: 25 de mayo (2010).

Gómez, E., L. Ríos y J. Peña, Madera, un Potencial Material Lignocelulósico para la Producción de Biocombustibles en Colombia, Información Tecnológica Vol. 23(6), 73-86 (2012)

Gupta, R. Alkaline pretreatment of biomass for ethanol production and understanding the factors influencing the cellulose hydrolysis, Tesis doctoral Auburn University, Alabama, USA (2008).

Hamelinck, C.N., G. Van Hooijdonk, y A. P. C. Faaij, Ethanol from lignocellulosic biomass: techno-economic performance in short-, middle- and long-term, Biomass Bioenergy 28 384-410 (2005). 
Kim, T. H. y Y. Y. Lee, Pretreatment of corn stover by soaking in aqueous ammonia at moderate temperatures, Appl Biochem Biotechnol 137-140(1-12):81-92 (2007).

Kropff, M. PROTA: Plant Resources of Tropical Africa. http://database.prota.org/publishedspeciesEn.htm (2000). Acceso: 25 de mayo (2010).

$\mathrm{Li}, \mathrm{X} ., \mathrm{T}$. Hyun y N. O. Nghiem, Bioresource Technology Bioethanol production from corn stover using aqueous ammonia pretreatment and two-phase simultaneous saccharification and fermentation (TPSSF), Bioresource Technology, 101(15), 5910-5916 (2010).

Marsden, W., P. Gray, y M. Quinlan, Evaluation of the DNS method for analysing lignocellulosic hydrolysate, J. Chem. Tech. Biotechnol., 32, 1016 (1982).

Mejía, L. F. y D. C. Alban, Hidrólisis y fermentación alcohólica simultánea (HFS) del residuo agroindustrial del mango común (Mangifera indica L) utilizando levaduras Saccharomyces cerevisiae spp y cepa recombinante RH 218, Universidad de San Buenaventura, Sede Cali Colombia. Revista Científica Guillermo de Ockham, Vol. 7, Núm. 2, julio-diciembre, pp. 51- 64 (2009).

Miller, GL. Use of Dinitrosalicylic acid reagent for determination of reducing sugars, Anal. Chem., 31, 426 (1959).

Monavari, S., M. Galbe, y G. Zacchi, Impact of impregnation time and chip size on sugar yield in pretreatment of softwood for ethanol production, Bioresource Technology 100 (24), 6312-6316 (2009).

Mosier, N., C. Wyman, B. Dale, R. Elander, Y. Y. Lee, M. Holtzapple, y M. Ladisch, Features of promising technologies for pretreatment of lignocellulosic biomass, Bioresource Technology 96 673-686 (2005).

Nguyen, Q. A., M. P. Tucker, B. L. Boynton, F. A. Keller y D. J. Schell, Dilute acid pretreatment of softwoods, Applied Biochemistry and Biotechnology 1998;70-72:77-87.

Pettersen, R, The chemistry of solid wood, in The chemical composition of wood by R. Rowell, pp 57-126, American Chemical Society, U.S. Department of Agriculture, USA (1984).

Saddler, J. N., L. P. Ramos y C. Breuil, Steam pretreatment of lignocellulosic residues. Bioconversion of Forest and Agricultural Residues, capítulo 3, ed. J. N. Saddler. CAB International, Oxford, UK, pp. 73-92 (1993).

Sánchez, Ó. y C. Cardona, Trends in biotechnological production of fuel ethanol from different feedstocks, Bioresource Technology 99 5270-5295 (2008).

Silverstein, R. A., Y. Chen, R. R. Sharma-shivappa, M. D. Boyette y J. Osborne, A comparison of chemical pretreatment methods for improving saccharification of cotton stalks, Bioresource Technology, 98, 30003011 (2007).

Söderström, J., M. Galbe y G. Zacchi, Effects of washing on yield in one- and two-step steam pretreatment of softwood for production of ethanol, Biotechnology Progress 20, 744-749 (2004).

Sun, Y. y J. Cheng, Hydrolysis of lignocellulosic materials for ethanol production: a review, Bioresour. Technol, 83, 11 (2002).

Unidad de Planeación Minero Energética. Plan Energético Nacional. Estrategia Energética Integral Visión 2003 - 2020. (2003).

Uribe, G. Pinus patula como base en la producción de pulpa para papel. Tesis de pregrado, Dpto. de Ingeniería Forestal, Universidad Nacional de Colombia. Medellín, Colombia (1967).

Varnero, M. T., M. S. Quiroz y C. H. Álvarez, Utilización de Residuos Forestales Lignocelulósicos para Producción del Hongo Ostra (Pleurotus ostreatus), Información Tecnológica Vol. - 21 № 2 (2010).

Vidal, P. y J. Molinier, Ozonolysis of lignin — Improvement of in vitro digestibility of poplar sawdust, Biomass Volume 16, Issue 1, Pages 1-17 (1988).

Zhang, W., y A. Geng, Improved ethanol production by a xylose-fermenting recombinant yeast strain constructed through a modified genome shuffling method, Biotechnology for biofuels, 5(1), 46 (2012). 